\title{
AEROACOUSTICS OF THE IMPELLER IN HOVERING MODE
}

\author{
a)Pavel Bulat, b)Pavel Chernyshov, c)Anton Kurnukhin, ${ }^{\text {d) }}$ Konstantin Volkov \\ a) Sevastopol State University, Sevastopol, Russia; BSTU "VOENMEH" named after D.F. Ustinov, St. Petersburg, Russia, \\ pavelbulat@mail.ru \\ b) Sevastopol State University, Sevastopol, Russia, pashachp8@gmail.com \\ c)Saint Petersburg Polytechnic university, St. Petersburg, Russia, anton.kurnukhin@outlook.com \\ d)Kingston University, London, United Kingdom, k.volkov@kingston.ac.uk
}

\begin{abstract}
The main role in reducing the noise generation of unmanned aerial vehicles is played by aeroacoustic improvement of their power plant. To identify the acoustic field generated by the rotation of the impeller, which is used on an unmanned aerial vehicle of a quadcopter type, numerical modeling was carried out with the identification of the turbulent structure of the flow using the Large Eddy Simulation (LES). To calculate the noise, the Fox Williams-Hawkings (FW-H) integral method was applied, which makes it possible to determine the aeroacoustic characteristics in the far flow field. Using this technique, the noise level at various points was determined, a spectral analysis of the noise was carried out, and the directional pattern of acoustic radiation was plotted for various speeds of rotation of the propeller in hovering mode.
\end{abstract}

Keywords: quadcopter, impeller, numerical modeling, aeroacoustics, directional pattern, eddy-resolving modeling

DOI: 10.36336/akustika20213916

\section{INTRODUCTION}

Quadcopter is an object with a small unobtrusive largest scattering cross section when a radar direction finding. From the point of view of practice, it is important to analyze the acoustic noise generated by the impeller and the aircraft as a whole [1]. Registration and processing of the acoustic signal is of interest for identifying an aircraft of this class and the maneuver it performs, as well as for extracting information useful for monitoring the location and movement parameters $[2,3]$.

To study the patterns of motion of a quadcopter in practice, a mathematical model is used that describes the motion of a rigid body with six degrees of freedom [4, 5]. The method for calculating the lift of a remotely piloted aircraft experiencing the influence of the ground effect is given in [6]. The aerodynamic characteristics of the impeller in the axial flow mode are determined in the work [7] based on the data of numerical simulation. In [8], at the preliminary stage of modeling, the rotor is represented in the form of an infinitely thin flat disk that maintains a pressure drop, simulating the creation of a lifting force by the rotor. Calculations are made based on Reynolds averaged Navier-Stokes equations.

Aeroacoustic rotor characteristics of the ring are in [9-18] using both experimental methods and simplified computational approaches and using modern numerical methods. The end parts of the propeller blade operate in the region of high Mach numbers, and a change in the geometry of this part of the blade leads to significant changes in the vortex structure of the forming flow and pressure field. In this case, the maximum of the broadband sound radiation near the rotor corresponds to the region of interaction of the blade with the tip vortex.
In [19], the flow around a six-bladed propeller is modeled using TVD schemes on a tetrahedral mesh, and the acoustic noise in the far field is calculated using the Fox Williams - Hawkings (FW-H) method. Modeling flow of the rotor in the general case of motion, as well as determination of its aerodynamic and acoustic characteristics are carried out in studies $[20,21]$. The developed software makes it possible to simulate the features of the flow around both an isolated main rotor with elastic blades making a swing motion, and together with the fuselage and the tail rotor [22-25]. In particular, Detached Eddy Simulation (DES) is used in [25] to solve the conjugate problem of calculating the aeroelastic deformation of propeller blades. The results of numerical calculations are used to refine the calculated vortex models and models for generating broadband noise.

In this paper, we simulate large eddies of a turbulent flow of a viscous compressible gas induced by the impellers of a quadcopter. Based on the calculated non-stationary pressure field, the sound pressure level in the far field is determined using the acoustic analogy method. Both the spectral analysis of the noise is carried out, and the integral directivity patterns of the acoustic radiation are constructed at different angular speeds of the rotor rotation. Different angular rotor speeds simulate vertical take-off and landing modes.

\section{NUMERICAL SIMULATION}

\subsection{Geometric model}

Highly loaded propellers $\mathrm{K} 6$ are considered using an air ring, which is a body of revolution with an aerodynamic profile in cross section (Fig. 1). The ring allows you to increase the speed in the plane of rotation of the propeller and the mass air flow 
rate, as well as create a circulation of speed around the profile of the ring, which leads to an increase in thrust, a decrease in power on the rotor blades and an increase in the profile efficiency of the blades. The combination of a screw and a ring forms a single impeller design. To design the propeller blades, a graph from [1] is used for the distribution of the relative thickness c, curvature $f$, rotation angle $\varphi$ and the length of the chord of the blade profile in plan $b$ along the relative radius of the propeller (the radius of the section plane relative to the radius of the entire propeller). The impeller geometry is shown in Fig. 2.

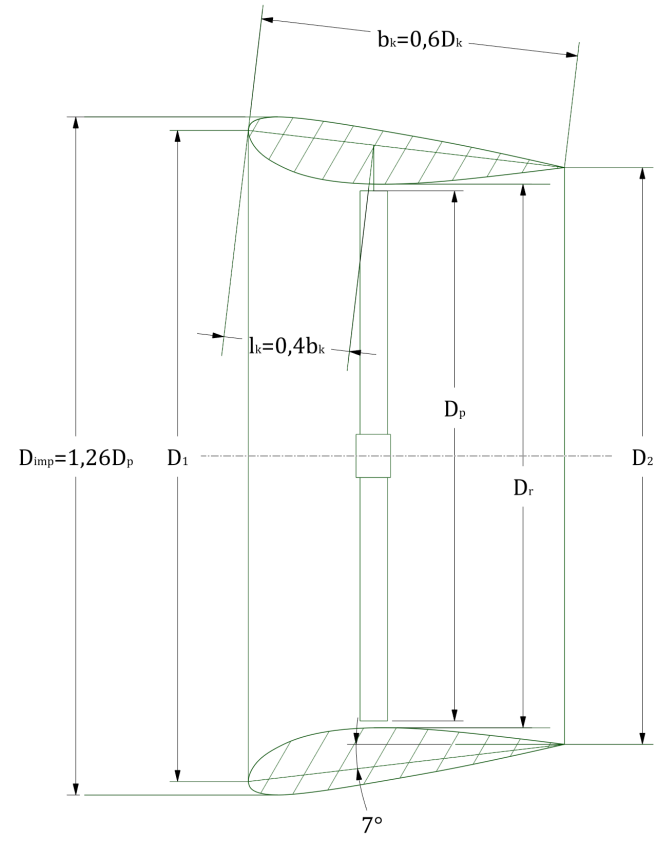

\section{Fig. 1: Impeller design sketch}

The choice of the K6 propeller is also due to the presence in the public domain of extensive studies of TsAGl, including the determination of the aerodynamic characteristics of the impeller and the effect on them of various design changes. The calculation parameter is the angular speed of the rotor rotation. The propeller geometrical parameters are calculated based on the geometry of the K6-VK1 impeller with a four-blade propeller, $25 \%$ shortened by the length of the air ring and a hub fairing. The propeller diameter is $760 \mathrm{~mm}$, and the ring outlet diameter is $780 \mathrm{~mm}$.

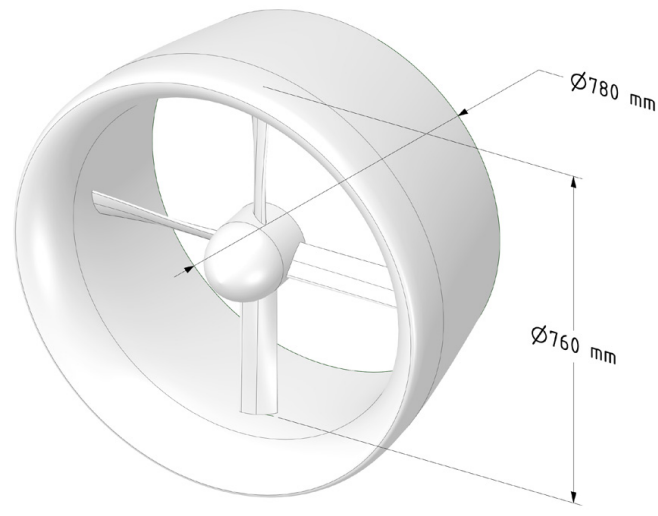

Fig. 2: Impeller geometry
The angle of the blades, determined by the angle of rotation of the blade profile, which is at a relative radius of 0.75 , was set at $28^{\circ}$. Such an angle was chosen in accordance with the effective operating mode of the propeller, which provides maximum thrust at a low incoming flow, that is, in conditions of vertical takeoff and landing.

\subsection{Mathematical Model and Boundary Conditions}

To simulate the flow induced by the rotation of the impeller blades of a quadcopter, the full Navier - Stokes equations are used, which describe the flow of a viscous compressible gas and are closed using the WALE subgrid viscosity model. Calculations are carried out to find non-stationary fields of physical variables that characterize the flow around the impeller and allow determining the aerodynamic loads on the rotor blades. The working medium is air. The air density changes according to the ideal gas model. Air viscosity is considered constant.

The calculation of the acoustic characteristics of the impeller is based on the method of acoustic analogy and the integral method of Fox Williams - Hawkings (Ffowcs Williams - Hawkings, FW - H).

The computational domain consists of rotating (rotor) and stationary (stator) subareas. To take into account the interaction between the regions and stitch the calculation results in each of the subdomains, the technology of sliding grids is used.

At the entrance to the computational domain, the speed and total temperature are set, at the other boundaries, the static pressure is indicated. The turbulence intensity at the inlet boundary is $5 \%$, and the ratio of the turbulent viscosity to the dynamic viscosity of the air is 10 . On the walls, the adhesion and no-flow conditions are applied. For temperature, the condition of zero heat flux is applied.

The calculations were performed on unstructured grid, consisting of polyhedral cells. The total number of cells is 3 million. The maximum characteristic cell size in the main part of the region is $0.1 \mathrm{~m}$. Part of the grid with prismatic cells adjoins the solid walls. The thickness of the prismatic layer is assumed to be $1 \mathrm{~mm}$, the number of layers is 5 , and the cell growth factor is 1.2 .

\subsection{Numerical method}

Time integration is carried out by the Runge - Kutta method of the third order of accuracy. Discretization of inviscid flows is carried out using the MUSCL scheme (Monotonic Upstream Schemes for Conservation Laws), discretization of viscous flows is carried out by the method of a centered scheme of the second order of accuracy. The MUSCL scheme allows one to increase the order of approximation in spatial variables without losing the monotonicity of the solution, satisfies the TVD (Total Variation Diminishing) condition, and is a combination of second-order accurate centered finite differences and a dissipative term. Finding the gradient and pseudo-Laplacian at the midpoint of the control volume face is based on ratios adapted for calculations on highly stretched meshes used in the boundary layer. The geometric multigrid method is used to solve the system of difference equations [26-27]. A system of grids of various resolutions is constructed using the Edge-Collapsing Method. 
Acoustic problem solution is found as the sum of the surface integral of a function of pressure and pulsation rate and spatial integral of the distribution function of the quadrupole sources. At low Mach numbers, quadrupole sources of sound are neglected, and the control surface of integration is identified with the surface of the streamlined body [25]. In this case, the acoustic field of the streamlined body is determined by the value of non-stationary dynamic loads acting on it from the flow side. Accounting surface integrals method substantially complicates the investigation of noise emission bodies of complex spatial configuration and reduces the performance of calculations.

The calculations are carried out with a time step of 5.10-6, which corresponds to the resolution of waves with a frequency of up to $50 \mathrm{kHz}$. The numerical calculation was carried out using the ANSYS Fluent software for numerical simulation of gas dynamics.

\subsection{Calculation results}

The results of aerodynamic calculations are used to simulate the propagation of acoustic disturbances in the near and far fields and to find the aeroacoustic characteristics of the impeller. It is assumed that the quadcopter is at a height of $15.3 \mathrm{~m}$ above a solid surface with the impeller orientation corresponding to vertical take-off and landing (the plane of rotation of the propeller is parallel to the solid surface). The side of the computational domain boundaries are located at a distance of $5 \mathrm{DB}$.

The mode of axial flow around the propeller is investigated, and numerical calculations are carried out at different angular speeds of rotation of the rotor (the main design version corresponds to the angular speed $\omega=3000 \mathrm{rpm}$ ). The oncoming flow velocity is assumed to be zero. The Reynolds number is calculated from the chord of the blade and its end speed, the Reynolds number at the end is $R e=5.8 \cdot 105$. Due to the symmetry of the rotor and the axial flow regime, not the entire propeller is modeled, but the sector with one blade with the setting of periodic boundary conditions on the meridional planes of the sector.

The use of a vortex-resolving approach to the description of turbulent flow makes it possible to resolve not only the structure of end vortices, but also the vortex sheet that comes off the blades, as well as the interaction of vortex structures of various scales. Closed areas of vorticity indicates the presence of discrete vortex bundles, which at some distance from the propeller are blurred, forming a continuous area of vorticity. The results of gas-dynamic calculations show that near a solid surface the speed of the thrown air flow is about $18 \mathrm{~m} / \mathrm{s}$. The air flow near the impeller is shown in Fig. 3.

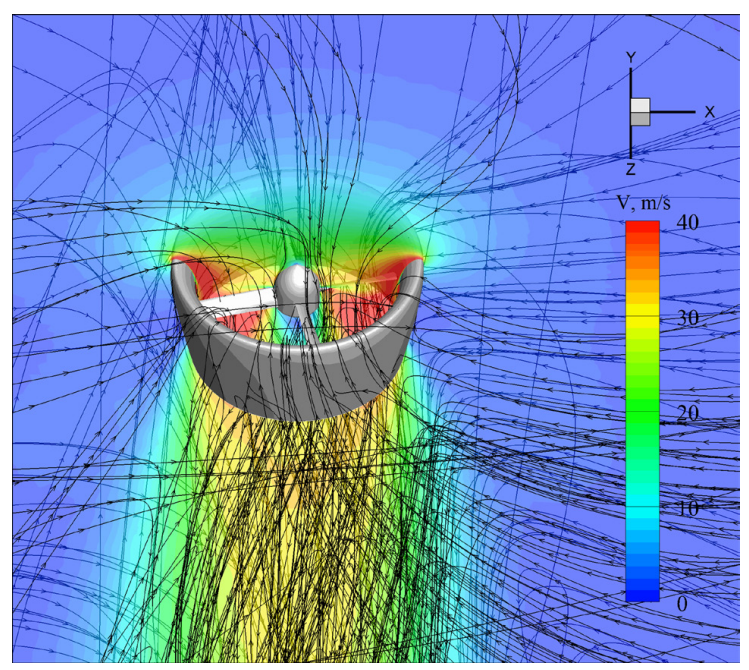

Fig. 3: Impeller flow velocity field

The time fluctuations of pressure obtained in the process of unsteady gas-dynamic calculation make it possible to determine the acoustic characteristics of the impeller both in the near and far fields. The most important characteristics are the dependence of the overall sound pressure level (OASPL) on the direction to the observation point, and the spectral composition of the acoustic signal at the observation points. The acoustic signal at the observation points is obtained as a result of data processing on the control surfaces after the calculation. The spectral characteristics of signals are found using methods based on the discrete Fourier transform.

At the stage of constructing the geometry of the computational domain, an array of points is selected at which the acoustic characteristics will be measured. The points are located in the axial direction with a step of $3.38 \mathrm{~m}$ and in the direction of removal from the axis of rotation with a step of $2 \mathrm{~m}$. The geometry of the computational domain with the considered points of space is shown in Fig. 4.

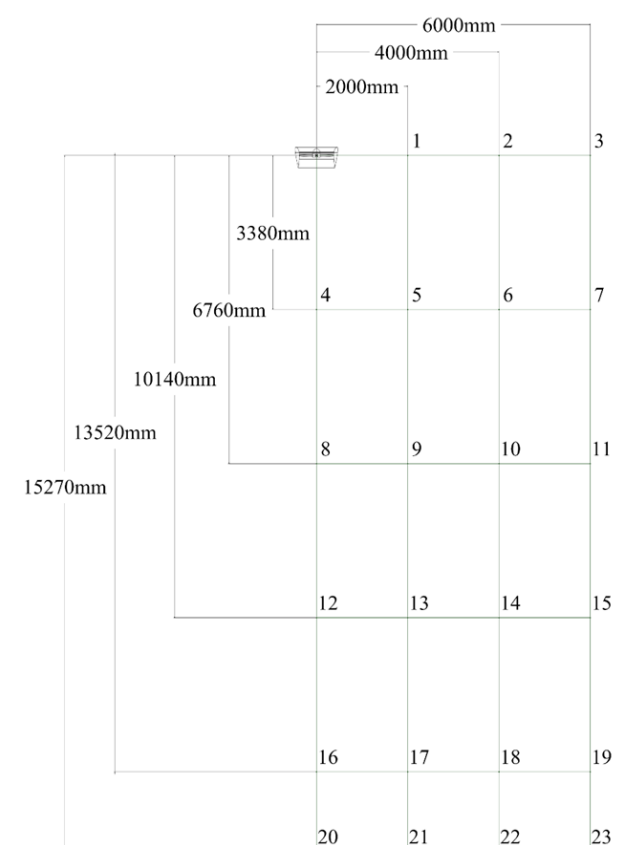

Fig. 4: Geometry of the computational domain with indication of the considered points in space 
Based on the results of the acoustic calculation, the values of the total noise level at the points under consideration (Fig. 5) and the distribution of the sound pressure level over the wave frequencies in each of them were obtained. Figure 6 shows the distributions of the sound pressure level over discrete frequencies at points 2 and 16 . Point 2 is located in the plane of rotation of the propeller, point 16 is located at a height of 1.75 $\mathrm{m}$ from the flat surface on the axis of rotation of the propeller. At point 16 (at the level of the average height of a person), the overall sound pressure level is about $72 \mathrm{~dB}$, which in many sources is classified as noise equivalent to a loud conversation.

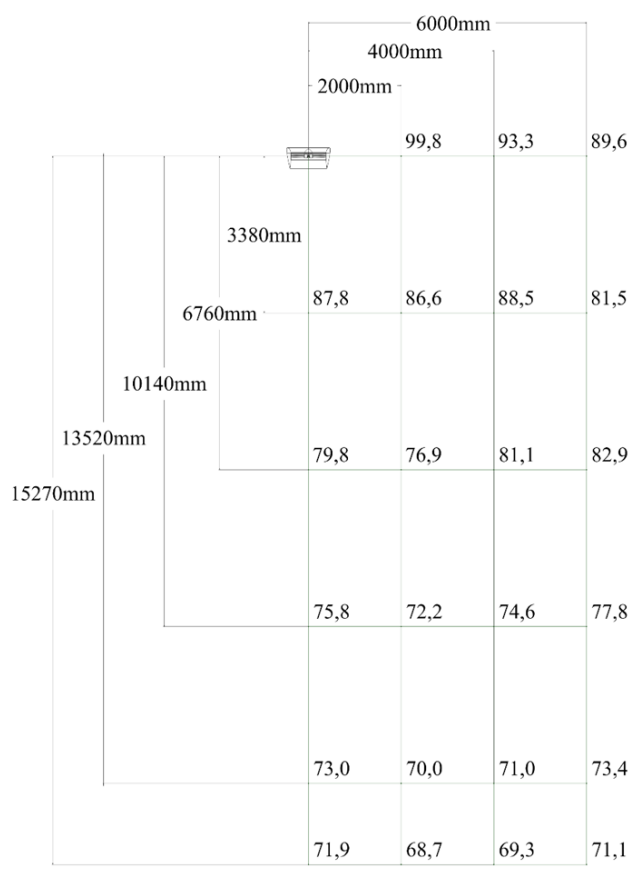

Fig. 5: Overall sound pressure level (OSPL) values at selected points $(d B)$

At the points located on the axis of rotation, three harmonics equidistant from each other are visible, corresponding to the frequencies of the discrete tone. The probable suppression of these harmonics by some change in the design of the propulsion unit would reduce the outgoing noise even more.

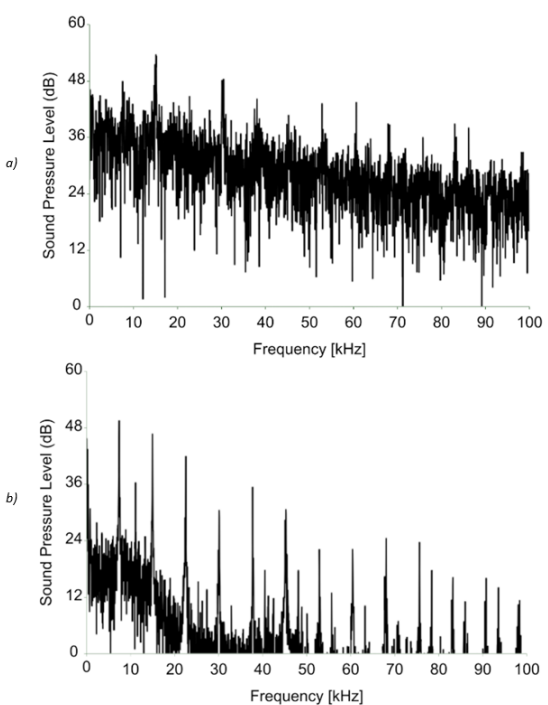

Fig. 6: Distribution of the sound pressure level at points 2 (a) and $16(b)$ over discrete frequencies $(d B)$
According to the results of calculation of unsteady aerodynamic OSPL level found on spherical surfaces around the impeller for different rotor speeds (see Fig. 7). To analyze the results, several control surfaces are selected, which are specified in the form of bodies of revolution with an axis coinciding with the axis of rotation of the propeller. The plane of the diagram, built in a polar coordinate system, is perpendicular to the plane of rotation of the propeller. Distance to control surfaces is $1,2,5$ and 10 meters. The sound pressure level is located at twenty points on an arc with a certain radius, after which the curve is constructed using spline interpolation.
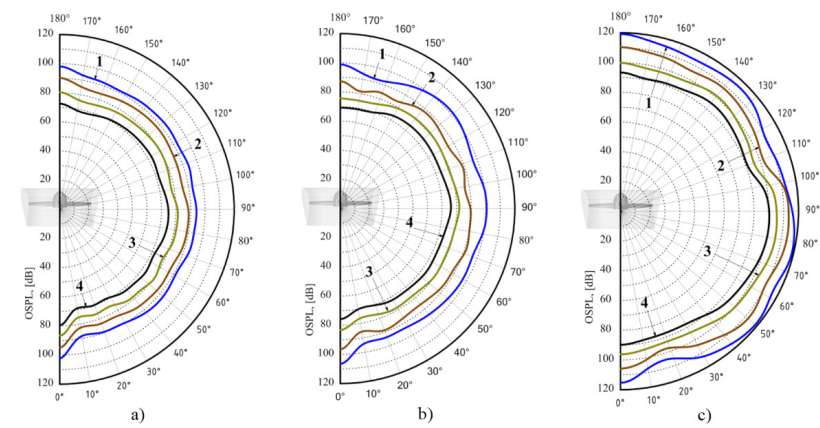

Fig. 7: Directional patterns of acoustic radiation on the control surfaces located at a distance of 1 (line 1), 2 (line 2), 5 (line 3) and 10 (line 4) $\mathrm{m}$ from the rotor rotation axis at $\omega=2000$ (a), 3000 (b) and 4500 (c) rpm

The maximum noise level generated by a rotating propeller is observed behind the ring in the plane of rotation of the propeller and near this plane. With distance from the propeller, the noise level decreases. With an increase in the speed of rotation of the propeller, the total power of sound radiation increases. The main sources of sound are the areas of separation of end vortices and the areas of their interaction with small-scale vortex structures.

The Proudman formula is used to estimate the power of broadband sound sources using time-averaged flow characteristics (Fig. 8). Such a distribution is rather uniform and is characterized by a monotonic increase in the size of the region in which the maximum generation of broadband noise is observed.
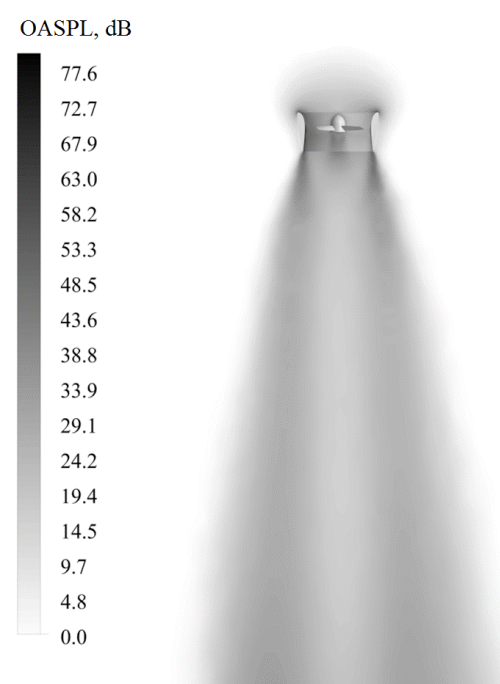

Fig. 8: Power distribution of broadband sound radiation sources using time-averaged flow characteristics 


\section{CONCLUSION}

Problem-oriented means of numerical simulation of the aerodynamic characteristics of the impeller in various operating modes have been created. Research and spectral analysis of the noise generated by the impeller at various operating modes has been carried out. The distribution diagrams of the sound pressure level in the far field were obtained by calculation. The nature of the spectrum and the location of the amplitude maxima can be useful for the identification and classification of aircraft based on noise direction finding data.

Applicable to the object under consideration, which is a propeller that creates thrust for the flight of an aircraft, the obtained level of acoustic radiation seems to be acceptable. Often, such aircraft create acoustic noise, which significantly limits their use in various conditions (in particular, in an urban environment). A fairly low level of generated noise was achieved by using highly loaded propellers, which make it possible to obtain a sufficiently large thrust force at a relatively low rotational speed.

\section{ACKNOWLEDGEMENT}

This work was financially supported by the Ministry of Science and Higher Education of the Russian Federation during the implementation of the project "Fundamental Foundations of Mechanics, Monitoring and Control Systems for Unmanned Aviation Systems with Shaping Structures Deeply Integrated with Power Plants, and Unique Properties Not Used Today in Manned Aviation" FEFM-2020-0001.

\section{REFERENCES}

[1] Ostroukhov S.P.: Aerodynamics of propellers and impellers. M.: Fizmatlit, 328 c., 2014

[2] Czyba R., Szafranski G.: Control structure impact on the flying performance of the multi-rotor VTOL platform - design, analysis and experimental validation, International Journal of Advanced Robotic Systems, Vol. 10. No. 1., p. 1-9., 2012

[3] Hrishikeshavan V., Chopra I.: Performance, flight testing of shrounded rotor micro air vehicle in edgewise gusts, Journal of Aircraft, Vol. 49., p. 193-205, 2012

[4] Popov N.I., Emelyanova O.V., Yacun S.F.: Simulating the dynamics of a quadcopter flight, Pozharnaya tekhnika, № 4(13), p. 69-75, 2014

[5] Kanatnikov A.N., Akopyan K.R.: Quadcopter flat motion control, Mathematics and mathematical modeling, Bauman Moscow State Technical University, № 2, p. 23-36, 2015

[6] Shaydakov V.I.: Influence of the proximity of the earth on the aerodynamic characteristics of an aircraft with a rotor-in-a-ring carrier system, Electronic journal „Trudy MAI", №. 49, p. 1-14, 2011

[7] Garipova L.I., Batrakov A.S., Kusymov A.N., Mikhaylov S.A., Barakos D.: Determination of the aerodynamic characteristics of the main rotor model in the axial flow mode, Proceedings of higher educational institutions. Aviation technology, № 3, p. 7-13, 2014

[8] Batrakov A.S., Garipova L.I., Kusymov A.N., Mikhaylov S.A., Barakos D.: The use of computational fluid mechanics in the problem of determining the aerodynamic characteristics of a helicopter, Scientific-methodical electronic journal "Concept", $t$. 20, p. 2471-2475, 2014

[9] Diizy F., Barakos D., Kusymov A.N., Kusymov S.A., Mikhaylov S.A.: DES-modeling of the helicopter main rotor flow, Proceedings of universities. Aviation technology, № 1, p. 40-46, 2018

[10] Rumsey CL, Biedron R., Farassat F., Spence P.: Ducted-fan engine acoustic predictions using a Navier-Stokes code, Journal of Sound and Vibration, Vol. 213, No. 4, p. 643-664, 1998

[11] Reboul G., Polacsek C., Lewy S., Heib S.: Aeroacoustic computation of ducted-fan broadband noise using LES data, Journal of Acoustic Society of America, Vol. 123, No. 5, p. 3539-3539, 2008

[12] Rhee W., Myers L., Mclaughlin D.: Statistical methods of proficiency testing processing and evaluation, Aeroacoustics of vertical lift ducted rotors, AIAA Paper, No. 2009-3333, 2009

[13] Astley R., Sugimoto R., Achunche I., Kewin M., Mustafi P., Deane E.: A review of CAA for fan duct propagation and radiation, with application to liner optimization, Procedia Engineering, Vol. 6, p. 143-152, 2010

[14] Yang Y., Veldhuis LLM, Eitelberg G.: Investigation of propeller induced ground vortices by numerical and experimental methods, AIAA Paper, NO. 2015-3302, 12 p., 2015

[15] Jiang Y., Zhang B., Huang T.: CFD study of an annular-ducted fan lift system for VTOL aircraft, Aerospace Journal, Vol. 2, p. $555-580,2015$

[16] Heinzen S. B., Hall CE, Gopalarthnam A.: Development and testing of a passive variable-pitch propeller, Journal of Aircraft, Vol. 52, p. 748-763, 2015

[17] Kutty H., Rajendran P.: 3D CFD simulation and experimental validation of small APC slow flyer propeller blade, Aerospace Journal, Vol. 4, p. 1-11, 2017

[18] Malgoezar A., Vieira A., Snellen M., Simons DG, Veldhuis LL: Experimental characterization of noise radiation from a ducted propeller of an unmanned aerial vehicle, International Journal of Aeroacoustics, 2019 
[19] Kopyev V.F., Titarev V.A., Belyaev I.V.: Development of a methodology for calculating the noise of propellers using supercomputers, TsAGI scientific notes, t. XLV, № 2, p. 78-106, 2014

[20] Costes M., Renaud T., Rodriguez B.: Rotorcraft simulations: a challenge for CFD, International Journal of Computational Fluid Dynamics, Vol. 26, No. 6-8, p. 383-405, 2012

[21] Costes M., Renaud T., Rodriguez B., Reboul G.: Application of vorticity confinement to rotor wake simulations, International Journal of Engineering Systems, Modelling and Simulation, Vol. 1, No. 1-2, p. 102-112, 2012

[22] Dehaeze F., Barakos GN: Hovering rotor computations using an aeroelastic blade model, The Aeronautical Journal, Vol. 116, No. 1180, p. 621-649, 2012

[23] Abalakin I.V., Bakhvalov P.A., Bobkov V.G., Kozubskaya T.K., Anikin V.A.: Numerical simulation of the aerodynamic and acoustic characteristics of a propeller in a ring, Math modeling, t. 27, № 10, p. 125-144, 2015

[24] Abalakin I.V., Anikin V.A., Bakhvalov P.A., Bobkov V.G., Kozubskaya T.K.: Numerical study of the aerodynamic and acoustic properties of a propeller in a ring, Izvestia RAN. Fluid and Gas Mechanics, № 3, p. 130-145, 2016

[25] Suvorov A.S., Korotin P.I., Sokov E.M.: Method of finite element modeling of noise emission generated by inhomogeneities of bodies moving in a turbulent fluid flow, Acoustic magazine, t. 64, № 6, p. 756-767, 2018

[26] Volkov K.: Numerical analysis of Navier-Stokes equations on unstructured meshes, Handbook on Navier-Stokes Equations: Theory and Analysis, D. Campos. Nova Science, p. 365-442, 2016

[27] Volkov K.: Multigrid and preconditioning techniques in CFD applications, CFD Techniques and Thermo-Mechanics Applications, Z. Driss, B. Necib, H.-C. Zhang, Springer International Publishing, p. 83-149, 2018

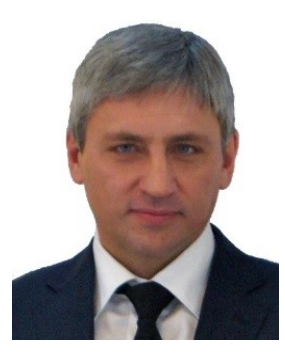

Pavel Bulat was born in 1966. Graduated from the Baltic State Technical University (BSTU) in 1993 and the Saint Petersburg State University of Economics and Finance in 2000. Doctor of Philosophy since 1998 and Doctor of Sciences since 2007. Doctor of Sciences in Physics and Mathematics since 2017 and Candidate of Economic Sciences. Specialist in the field of rocketry, aerodynamics of aircraft, supersonic aerogasdynamics, physics of shock-wave and detonation processes. Author of 24 patents in the field of aviation, engines and power plants. Deputy Co-Head of Aeronet Working Group on Science and Technology since 2017. Head of Aeronet Technologies subgroup since 2017. Head of the Aeronet Expert Council. Skolkovo expert in the Cosmos, Energotech, and Yadertech clusters. RUSNANO's expert in the following areas: energy efficiency, aerospace engineering, special electronics, MEMS, microelectronics, motors, material hardening, precision and special mechanics. Expert of the "Fund for the Promotion of Innovations". Author of over 150 publications. Chief Researcher of the Laboratory "Flight dynamics and control of unmanned aircraft systems" of the Sevastopol State University.

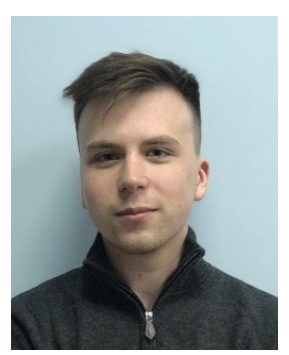

Pavel Chernyshov was born in 1996. Graduate student of Department of Plasma and Gas Dynamics and Heating Engineering of the Baltic State Technical University (BSTU). Junior Researcher, Flight Dynamics and Control of Unmanned Aviation Systems Laboratory, Sevastopol State University.

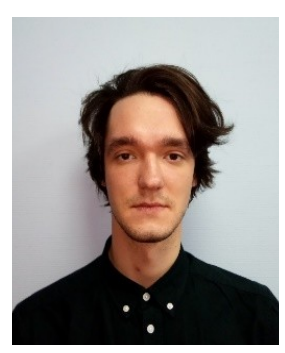

Anton Kurnukhin was born in 1996. Graduate student of Department of Plasma and Gas Dynamics and Heating Engineering of the Baltic State Technical University (BSTU). Junior Researcher, Flight Dynamics and Control of Unmanned Aviation Systems Laboratory, Sevastopol State University.

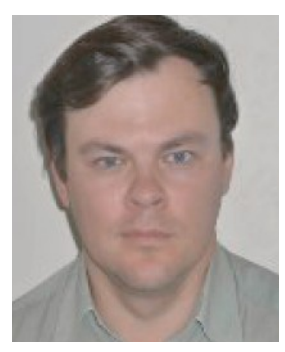

Konstantin Volkov was born in 1973. Graduated from the Baltic State Technical University (BSTU) in 1996 and the Saint Petersburg State University in 1997. Doctor of Philosophy since 1998 and Doctor of Sciences since 2007. Worked in BSTU as a research assistant from 1996 to 2002, from 1998 to 2002 as a lecturer, then a senior lecturer, at the Department of Plasma and Gas Dynamics and Thermal Engineering. Worked in the United Kingdom at the University of Central Lancashire from 2002 to 2004 and University of Surrey from 2004 to 2009. From 2009 works and lectures in the Kingston University in London. During his work in BSTU, took part in development and achievement of models designed for the description of intra-chamber processes in solid rocket motors. Author of over 170 academic papers and 10 books. Member of the Institute of Physics and the Institution of Mechanical Engineers (United Kingdom). 